\title{
Noteninflation an deutschen Hochschulen
}

\author{
Keine Trendwende in Sicht
}

Die Tendenz zu besseren Benotungen von Studienabschlussprüfungen an deutschen Hochschulen hat sich in den Jahren von 2015 bis 2019 weitgehend ungebrochen fortgesetzt. Besonders auffällig in den letzten Jahren ist eine deutliche Verschiebung weg von der Benotungsstufe "gut" hin zur Benotungsstufe "sehr gut". Verbesserte Benotungen sind in allen Fächern zu beobachten, wenngleich mit unterschiedlicher Dynamik. Die erheblichen Abweichungen im Niveau weisen aber auf immer noch sehr unterschiedliche Benotungskulturen in den untersuchten Fächern hin.

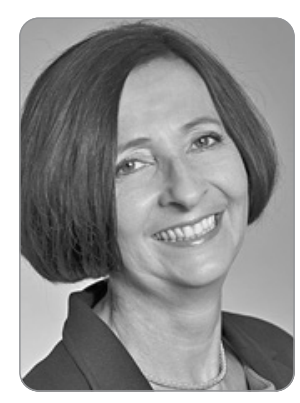

\section{Prof. Dr. Christa Wehner}

ist seit 2000 Studiendekanin Marktforschung und Konsumentenpsychologie an der Fakultät für Wirtschaft und Recht der Hochschule Pforzheim. Bevorzugte Forschungsgebiete: Qualitätsmanagement, Kundenzufriedenheitsforschung, Evaluation.

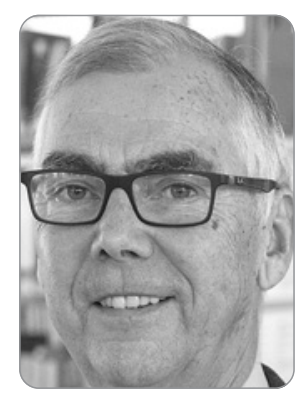

\section{Prof. Dr. Helmut Wienert}

war bis 2013 Professor für Volkswirtschaftslehre an der Fakultät für Wirtschaft und Recht an der Hochschule Pforzheim. Seine bevorzugten Forschungsgebiete sind: Bildungsökonomie, Empirische Wirtschaftsforschung.

In den letzten Jahren haben sich die Durchschnittsnoten von Schülern und Studenten in Deutschland im Trend deutlich verbessert. Da vielfach vermutet wird, dass deren Leistungen zumindest nicht im gleichen Umfang mitgewachsen sind, hat sich dafür der Begriff „Noteninflation“ eingebürgert. Leistungsmessungen finden nicht nur in der Ausbildung, sondern in fast allen Lebensbereichen statt, denn die Frage: "Wie gut bin ich im Vergleich zu anderen und was folgt daraus für mich?" stellt sich in unterschiedlicher Form immer wieder. Im Wirtschaftsleben geht es dabei um erbrachte Leistungen als Arbeitnehmer oder als selbständiger Anbieter auf einem Markt und damit um Marktpartizipationschancen und Einkommensansprüche. In vielen Fällen setzt Marktteilnahme fachliche Ausbildung voraus, und da- bei gibt es vielfältige Kontrollpunkte, an denen der Ausbildungsfortschritt gemessen wird, sei es durch Benotungen einzelner Prüfungsleistungen in Ausbildungsabschnitten oder durch abschließende Testierung von Fähigkeiten beim Ausbildungsabschluss.

Damit solche Beurteilungen einen Marktwert haben, muss die testierende Institution eine hinreichende Reputation haben. Nur wenn sich Dritte auf deren Urteil verlassen können, werden sie kostspielige eigene Leistungsmessungen unterlassen. In der Praxis sind natürlich Mischformen zu erwarten: Geht es um die Einstellung eines neuen Mitarbeiters in ein Unternehmen, so wird in der Regel eine bestimmte formale Mindestqualifikation vorausgesetzt werden, die durch Nachweise erbracht werden muss, die andere Institutionen (Schulen, Universitäten, Ausbildungsbetriebe, frühere Arbeitgeber etc.) ausgestellt haben. Auf dieser Basis wird eine "Vorsortierung" vorgenommen und die endgültige Auswahl erfolgt dann durch unternehmenseigene Beurteilungsprozesse. Je präziser (glaubwürdiger) die Beurteilungen durch Externe sind, umso geringer wird der unternehmensinterne Aufwand sein, und umgekehrt gilt natürlich auch: Je schwächer das "Signal“ der Externen bezüglich der tatsächlichen Qualität der Bewerber ist, umso mehr muss das Unternehmen in eigene Leistungsbeurteilungsprozesse investieren.

In einer früheren Untersuchung (vgl. Wienert, 2018) wurde gezeigt, dass sich die Notengebung bei Abschlussarbeiten an deutschen Hochschulen bis ins Jahr 2015 hinein in zunehmendem Maße auf die Notenstufen "gut" oder "sehr gut" verengt hat, und es wurde argumentiert, dass dies letztlich die Selektionsqualität der Zeugnisse beschädigt. Da inzwischen Daten für vier weitere Jahre vorliegen, wird 


\begin{tabular}{|c|c|c|c|c|c|c|c|c|}
\hline \multirow{2}{*}{ Fach } & \multicolumn{2}{|c|}{ Prüfungen } & \multicolumn{3}{|c|}{$\begin{array}{l}\text { Anteil Notenstufen } \\
2019\end{array}$} & \multicolumn{3}{|c|}{$\Delta$ in $\%$ vs. 2015} \\
\hline & 2019 & $\begin{array}{r}\Delta \text { in } \% \\
\text { vs } 2015\end{array}$ & $\begin{array}{l}\text { sehr } \\
\text { gut }\end{array}$ & gut & $\sum$ & $\begin{array}{c}\text { sehr } \\
\text { gut }\end{array}$ & gut & $\sum$ \\
\hline Germanistik & 14265 & $-1,0$ & 28,9 & 59,6 & 88,6 & 6,3 & $-3,2$ & 3,1 \\
\hline Anglistik & 7891 & $-4,5$ & 28,5 & 59,7 & 88,2 & 8,0 & $-5,9$ & 2,1 \\
\hline Psychologie & 15895 & 48,1 & 48,9 & 46,3 & 95,2 & 3,7 & $-2,6$ & 1,1 \\
\hline Sport & 5090 & $-1,5$ & 23,1 & 63,3 & 86,4 & 8,0 & $-7,2$ & 0,8 \\
\hline Politikwissenschaft & 5122 & 7,3 & 37,4 & 55,5 & 92,9 & 9,8 & $-7,6$ & 2,2 \\
\hline Sozialwissenschaft & 7120 & 1,4 & 32,3 & 57,6 & 89,9 & 7,4 & $-4,5$ & 2,9 \\
\hline Rechtswissenschaft & 17639 & 15,1 & 11,7 & 24,0 & 35,7 & 1,5 & $-0,4$ & 1,1 \\
\hline Verwaltungswissenschaft & 15715 & 28,5 & 6,7 & 51,9 & 58,6 & 2,4 & 1,7 & 4,1 \\
\hline Wirtschaftswissenschaft & 88781 & 4,2 & 22,2 & 60,8 & 83,1 & 9,2 & $-3,7$ & 5,5 \\
\hline Wirtschaftsingenieurwesen & 20032 & 3,0 & 24,3 & 62,1 & 86,4 & 11,0 & $-2,1$ & 8,9 \\
\hline Mathematik & 9973 & $-6,2$ & 32,4 & 50,6 & 83,0 & 6,0 & $-2,2$ & 3,8 \\
\hline Informatik & 28909 & 17,1 & 28,5 & 56,9 & 85,4 & 7,3 & $-3,6$ & 3,7 \\
\hline Physik & 7820 & $-4,2$ & 54,2 & 37,9 & 92,1 & 5,9 & $-3,0$ & 2,9 \\
\hline Chemie & 11108 & 2,9 & 45,5 & 44,4 & 89,9 & 3,0 & $-1,2$ & 1,8 \\
\hline Biologie & 14330 & $-4,0$ & 42,7 & 48,0 & 90,6 & 4,3 & $-3,2$ & 1,1 \\
\hline Humanmedizin & 17714 & 10,6 & 32,3 & 46,5 & 78,8 & 2,4 & $-0,2$ & 2,2 \\
\hline Maschinenbau & 36040 & $-6,5$ & 24,9 & 56,6 & 81,5 & 6,2 & $-2,5$ & 3,7 \\
\hline Elektrotechnik & 14775 & $-2,4$ & 28,4 & 54,5 & 82,9 & 5,9 & $-1,5$ & 4,4 \\
\hline Bauingenieurswesen & 10623 & 6,2 & 17,6 & 56,8 & 74,5 & 6,5 & 2,8 & 9,3 \\
\hline Kunstwissenschaft & 17534 & 2,0 & 61,8 & 35,7 & 97,5 & 13,4 & $-11,9$ & 1,5 \\
\hline Erziehungswissenschaft & 19943 & 20,7 & 40,5 & 54,0 & 94,5 & 13,6 & $-8,4$ & 5,2 \\
\hline Sozialwesen & 17457 & 17,3 & 37,8 & 57,4 & 95,2 & 12,3 & $-9,6$ & 2,7 \\
\hline Architektur & 9109 & 7,3 & 32,4 & 61,2 & 93,6 & 15,9 & $-10,6$ & 5,3 \\
\hline Summe dieser Fächer & 412885 & 6,3 & 29,9 & 53,6 & 83,4 & 7,7 & $-3,8$ & 3,9 \\
\hline
\end{tabular}

Tab. 1: Bestandene Abschlussprüfungen nach Fächern und Notenstufen an deutschen Hochschulen, 2019 und Veränderungen gegenüber 2015. im Folgenden geprüft, ob der Trend zu immer besseren Noten bis 2019 angehalten hat. Behandelt werden ausschließlich empirische Fragen, für eine Literaturübersicht und die Diskussion möglicher Gründe für die Noteninflation wird wieder auf Müller-Benedikt/Grözinger (Hrsg., 2017) verwiesen.

Um die Darstellung übersichtlich zu halten beschränkt sich Tab. 1 auf die an der Zahl der bestandenen Abschlussprüfungen gemessen wichtigsten Fächer. Datenquelle ist die Hochschulstatistik des Statistischen Bundesamtes (vgl. Destatis, 2020), wobei das dort verwendete Prüfungsjahr jeweils aus der Summe der abgelegten Prüfungen im Laufe des Wintersemesters und des folgenden Sommersemesters besteht, für das Jahr 2019 also aus dem Wintersemester 2018/2019 und dem Sommersemester 2019. Als Vergleichsjahr wird 2015 herangezogen.

Die Zahl der bestandenen Abschlussprüfungen ist vom Jahr 2015 bis zum Jahr 2019 in den in Tab. 1 erfassten Fächern insgesamt um 6,3 \% bzw. 1,5\% je Jahr gestiegen, und damit nur geringfügig stärker als die Zahl der Studierenden in diesem Zeitraum. In den Jahren 2003 bis 2015 war dagegen noch ein Anstieg der bestandenen Prüfungen um 6,6 \% je Jahr verzeichnet worden - weit mehr als der Anstieg der Studentenzahlen in dieser Zeit. Ursächlich dafür dürfte u.a. sein, dass der Übergang vom Diplom zu den gestuften Abschlüssen Bachelor bzw. Master (Bologna-Reform) inzwischen vollständig abgeschlossen ist.

Den mit der Umstellung verbundenen Effekt kann man beispielhaft wie folgt aufzeigen: Angenommen 1000 Studenten starteten jedes Semester in einen Diplom-Studiengang, der acht Semester umfasst. Der Einfachheit halber sollen alle ordnungsgemäß studieren, so dass jedes Semester ebenfalls 1000 Studenten abschließen. Durch die Bologna-Reform sollen im Umstellungssemester keine neuen DiplomStudenten mehr aufgenommen werden, sondern nun 1000 Studenten ein sechs-semestriges Bachelor-Studium beginnen. Sieben Semester lang werden dann noch 1000 DiplomAbschlüsse erfolgen, während die Bachelor-Anfänger nach sechs Semestern ebenfalls mit 1000 abschließen, sodass wegen der unterschiedlichen Studiendauer zwei Semester 


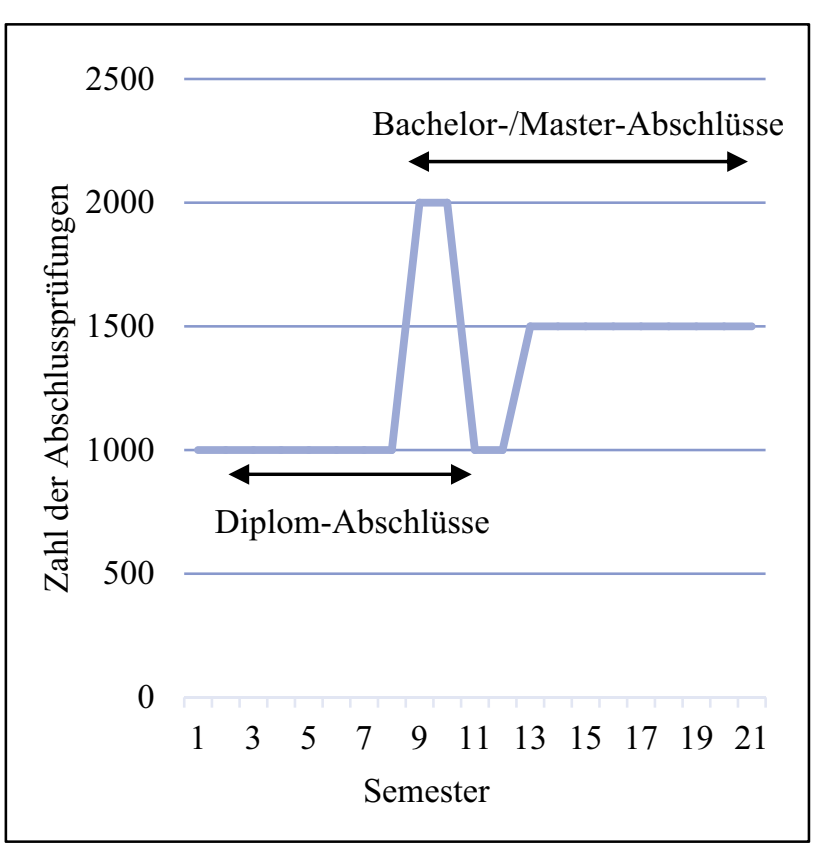

Abb. 1: Schematischer Verlauf der Zahl der Abschlussprüfungen beim Umstieg vom Diplom auf Bachelor/Master

lang 2000 statt 1000 Abschlüsse zu verzeichnen sind. Wenn zudem z.B. die Hälfte der ersten Bachelor-Absolventen ein Master-Studium anschließt, starten sieben Semester nach Umstellungsbeginn jeweils 500 Studenten, die nach vier weiteren Semestern abschließen. Insgesamt ergibt sich dann wegen der unterschiedlichen Studiendauer und der „Doppelgraduierung" der in Abb. 1 dargestellte Verlauf mit vorübergehenden Schwankungen bei langfristig höherem Niveau.

Der zwischen den Jahren 2015 und 2019 erfolgte Anstieg der bestandenen Prüfungen verteilt sich naturgemäß nicht gleichmäßig auf die verschiedenen Fächer. Besonders starke Zunahmen der bestandenen Prüfungen zeigen sich in so unterschiedlichen Bereichen wie Psychologie und Informatik, daneben sind deutliche Anstiege in Fächern, die vor allem staatliche Bedarfe befriedigen (Verwaltungswissen- schaften, Erziehungswissenschaften und Sozialwesen) zu verzeichnen. Bei den Fächern mit rückläufigen Prüfungszahlen sind vor allem Maschinenbau, Mathematik, Physik und Biologie auffällig.

Bei den Noten unterscheidet das Statistische Bundesamt die Notenstufen "mit Auszeichnung", "sehr gut", "gut", "befriedigend" und "ausreichend“. Da "mit Auszeichnung” nur in wenigen Fächern und bei der Promotion abgeschlossen werden kann, werden diese Bewertungen hier der Einfachheit halber der Notenstufe "sehr gut" zugeordnet. Im Jahr 2015 waren 22,2 \% aller bestandenen Prüfungen in den in Tab. 1 erfassten Fächern mit "sehr gut" bewertet worden, im Jahr 2019 waren es mit 29,9\% dagegen deutlich mehr. Die entsprechenden Werte für die Notenstufe "gut" betrugen 57,4\% im Jahr 2015 und 53,6\% in Jahr 2019. Der Rückgang der "gut“-Bewertungen fällt folglich deutlich geringer aus als der Anstieg der "sehr gut"-Noten - fasst man beide Kategorien zusammen, so ergab sich ein Anstieg der mit "gut oder besser" bewertete Anteile, und zwar von 79,6 auf 83,4\%.

Der Vergleich zweier Stichjahre kann durch Sondereinflüsse verzerrt sein, so dass es sich empfiehlt, die Tendenz mehrerer Jahre zur Kontrolle heranzuziehen. Aus Abb. 2 ist die Entwicklung des zusammengefassten Notenstufenanteils "sehr gut" der in Tab. 1 erfassten Fächer im Verlauf von 2011 bis 2019 ersichtlich. Es zeigt sich, dass in den Jahren von 2011 bis 2015 bei den "sehr gut"-Benotungen weitgehend Stagnation herrschte (was auch für die "gut"-Benotungen zutrifft), danach aber ein deutlicher Anstieg erfolgte, und zwar vor allem in den Jahren 2017 bis 2019. Dieses Grundmuster findet sich zwar nicht in allen, aber doch den meisten Fächern wieder, beispielhaft sind in $A b b .2$ Entwicklungen auf unterschiedlichen Niveaus für die Fächer Politikwissenschaft, Wirtschaftswissenschaft, Architektur und Verwaltungswissenschaft eingezeichnet worden. Der Trend zu besseren Noten hat also nach einer vorüber-
Quelle: Eigene Berechnungen nach Angaben in Statistisches Bundesamt, Fachserie 11, R. 4.2

Abb. 2: Anteil der Notenstufe "sehr gut" an allen bestandenen Abschlussprüfungen, 2011 bis 2019.

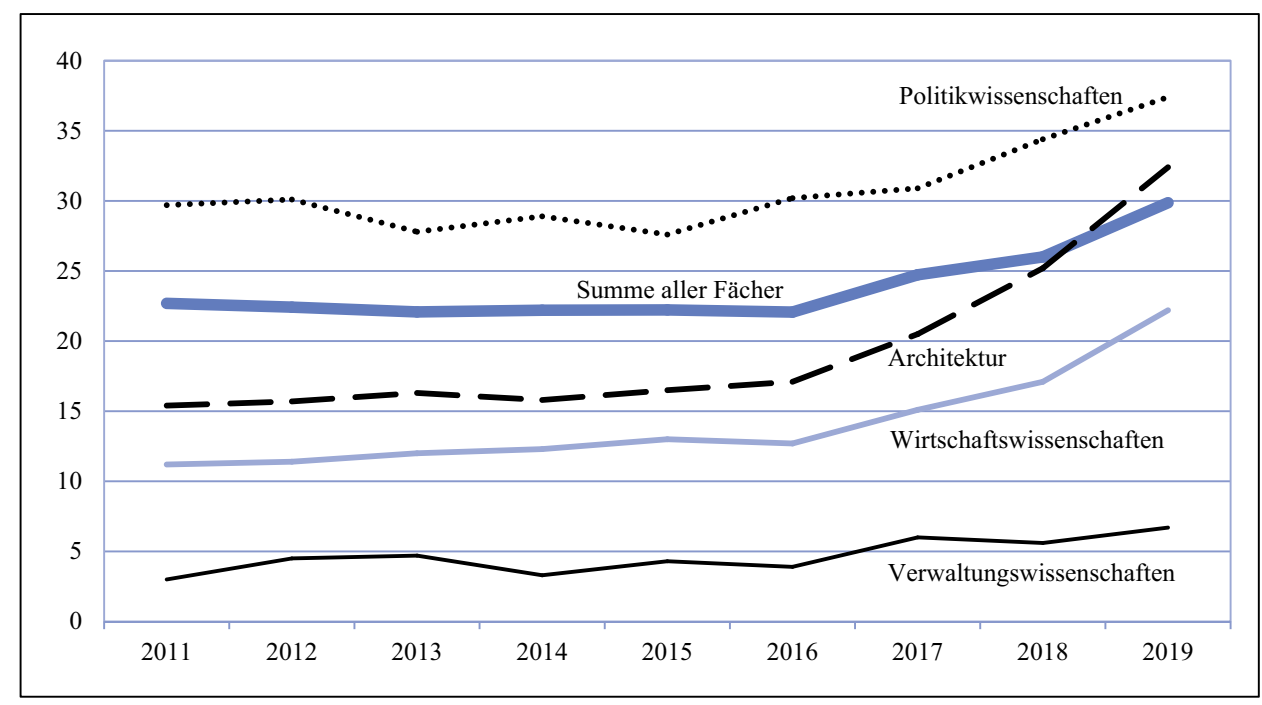




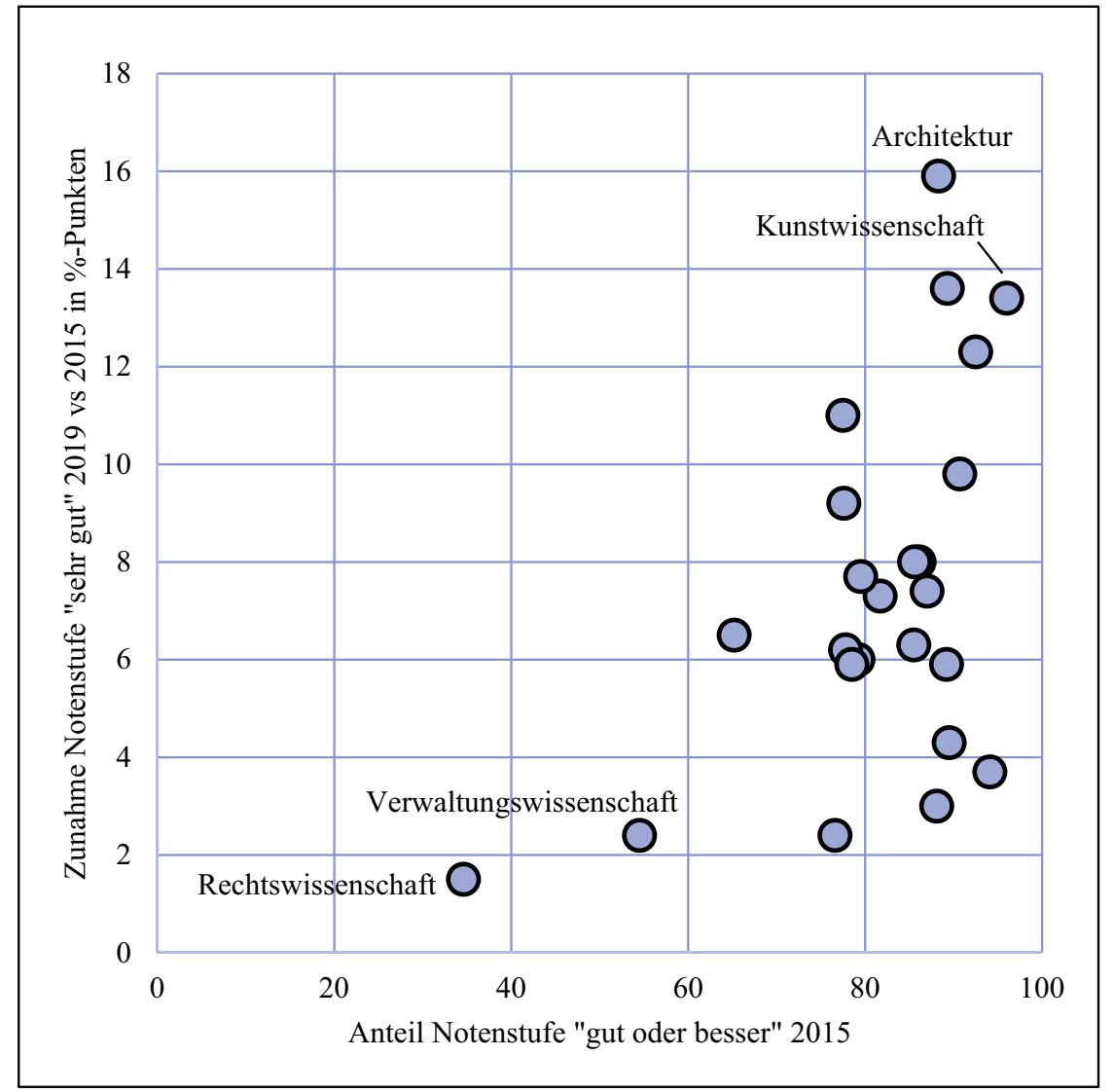

Quelle: Eigene Berechnungen nach Angaben in Statistisches Bundesamt, Fachserie 11, R. 4.2

Abb. 3: Zuwachs der Note "sehr gut" im Vergleich zum Ausgangsniveau der Notenstufe „gut oder besser" für 22 Fächer. gehenden Pause wieder Fahrt aufgenommen. Da die „Luft nach oben" bei schon zuvor kaum noch vergebenen Notenstufen "befriedigend" oder "ausreichend“ naturgemäß „dünn“ war, blieb als Ausweg zu besseren Durchschnittsnoten nur die Verschiebung der Anteile von "gut" nach "sehr gut", und genau diese Tendenz zeigen die aktuellen Zahlen.

In allen 22 erfassten Fächern steigt der Anteil der Notenstufe "sehr gut" im Vergleich der Jahre 2019 und 2015 an, und zwar in der Tendenz umso stärker, je höher der Anteil "gut oder besser“ im Jahr 2015 schon war (vgl. Abb. 3). Als Extreme sind die Fächer Architektur und Kunstwissenschaften zu nennen: Hier war der Anteil von "gut oder besser" schon 2015 deutlich über $90 \%$, und es fand eine Verschiebung von rund $15 \%$-Punkten weg von "gut" hin zu "sehr gut“ statt. Als Fächer mit moderaten Durchschnittsnoten und geringen Zuwächsen in der Notenstufe "sehr gut" verbleiben in Abb. 3 erkennbar allein noch die Rechtswissenschaft und die Verwaltungswissenschaft - möglicherweise, weil hier die Notengebung nicht allein in der Hand der Hochschulen liegt.

Es ist auffällig, dass der Anstieg der Anteile der Notenstufe "sehr gut" in allen Fächern stärker ist als der Rückgang der Anteile der Notenstufe "gut". Wie stark dies auf die Durchschnittsnoten durchschlagen kann zeigt folgende Beispielrechnung: Nimmt man der Einfachheit halber an, dass die Notenstufe "sehr gut" mit 1,0 erfasst wird, die No- tenstufe "gut" im Mittel mit 2,0 und die beiden restlichen Notenstufen "befriedigend" und "ausreichend" mit 3,5, so errechnet sich für 2015 bei den dort gegebenen Verteilungen für die Summe aller Fächer nach Tab. 1 eine Durchschnittsnote von 2,08. Durch die Verschiebungen zugunsten von "sehr gut" ergibt der gleiche Rechengang für das Jahr 2019 einen Durchschnittswert von 1,95. Für nur vier Jahre ist das eine beachtliche Verschiebung - hielte sie in dieser Form an, so würde die Durchschnittsnote relativ rasch auf eine gerundete 1,5 und damit die Notenstufe "sehr gut" zusteuern.

Notorisch hohe Werte von Anteilen der Notenstufen "gut oder besser" von 90 \% oder sogar darüber hinaus weisen die Fächer Psychologie, Politikwissenschaft, Sozialwissenschaft, Erziehungswissenschaft, Sozialwesen, Architektur, aber auch Physik, Chemie und Biologie auf. An der Spitze stehen die Kunstwissenschaften, in denen im Jahr 2019 stolze 97,5\% der Abschlussnoten im Bereich "gut oder besser" vergeben wurden, darunter allein knapp $62 \%$ in der Notenstufe "sehr gut". Relativ niedrige Durchschnittsnoten findet man wie schon erwähnt bei der Verwaltungswissenschaft und der Rechtswissenschaft.

Die Wirtschaftswissenschaft liegt mit ihren Abschlussnoten im oberen Mittelfeld, allerdings ist der Anstieg der Notenstufe "sehr gut" von $13 \%$ im Jahr 2015 auf 22,2 \% im Jahr 2019 sehr ausgeprägt gewesen; die Noten im Bereich "gut oder besser" sind damit von 77,5 auf $83 \%$ angewach- 
sen. Wie in Wienert (2018) seien für die Wirtschaftswissenschaft auch die Teilentwicklungen bei Promotionen, Lehramts- und Bachelor-/Master-Abschlüssen genannt: Bei den Promotionen ist der Anteil "mit Auszeichnung" von 34,1 \% im Jahr 2015 auf 31,7 \% zurückgegangen. Dies ist bemerkenswert, weil es dem bisherigen Trend widerspricht, und es ging zudem einher mit einem Rückgang der Zahl der Geprüften von 1401 auf 1249. Gleichzeitig stieg der Anteil der "sehr gut"-Bewertungen allerdings von 47,8 auf $51,9 \%$, so dass sich der Trend für die Summe dieser beiden Bestnoten von 81,9 auf 83,6 \% weiter fortsetzte. Bei den Lehramtsabschlüssen im Bereich Wirtschaftswissenschaften stieg der Anteil der Notenstufe "gut oder besser" im gleichen Zeitraum sehr kräftig von 80,3 auf 85,7 \% - im Jahr 2003 hatte er noch bei $64 \%$ gelegen.

Die Zahl der erfolgreichen Bachelor-Abschlüsse in Wirtschaftswissenschaft stagnierte zwischen 2015 und 2019 bei rund 56 000, der Anteil, der mit "sehr gut" abgeschlossen hat, verdoppelte sich von 7,7 auf 15,3\%, der der Notenstufe "gut" nahm geringfügig von 64 auf $63 \%$ ab, so dass der zusammengefasste Anteil deutlich stieg. Bei den MasterAbschlüssen gab es einen Anstieg der Absolventenzahlen von rund 24000 auf knapp 30 000; der Anteil der "sehr gut"-Abschlüsse hat sich mehr als verdoppelt (Anstieg von 15,3 auf $33 \%$ ), der der Notenstufe "gut" ging zwar zugleich deutlich zurück (von 69 auf $59 \%$ ), in der Summe bedeutet dies aber, dass rund $92 \%$ aller Absolventen den
Master mit der Notenstufe "gut oder besser" abschließen, nach rund $90 \%$ vier Jahre zuvor. Bemerkenswert ist auch die Entwicklung der Relation von Master- zu Bachelor-Absolventen: Sie nahm von 43 auf 53 \% zu. Der Bachelor verliert als erster berufsqualifizierender Abschluss offenbar rasch an Wert, bei den Masterabschlüssen gilt der Tendenz nach das gleiche für Abschlüsse mit der Notenstufe "gut". Auf der Suche nach Gründen für die in Tab. 1 verzeichneten erheblichen strukturellen Abweichungen der Anteile der Notenstufen zwischen den verschiedenen Fächern wird man aus den vom Statistischen Bundesamt in der Hochschulstatistik veröffentlichten Daten nicht fündig. Man könnte z.B. vermuten, dass besonders "schwere“ Fächer hohe Abbruchquoten mit niedrigen Durchschnittsnoten aufweisen. Nimmt man den Anteil endgültig nicht bestandener Abschlussprüfungen an allen Abschlussprüfungen als Indikator für "schwere Fächer" und den Anteil der Notenstufe "sehr gut" als Indikator für gute Durchschnittsnoten, so zeigt sich empirisch keinerlei Zusammenhang (vgl. Abb. 4).

Eine "sehr gut“-Quote von über $50 \%$ kann danach mit "Abbruchquoten" von $8 \%$ (Physik) oder auch nur $1 \%$ (Kunstwissenschaft) einhergehen, und auf der anderen Seite kann eine "sehr gut"-Quote von rund $30 \%$ mit „Abbruchquoten" von $10 \%$ (Informatik), $4 \%$ (Germanistik) oder 0,3\% (Humanmedizin) verbunden sein. Erwähnenswert ist, dass die Abbruchquote eine deutlich steigende Tendenz aufweist: Im
Quelle: Eigene Berechnungen nach Angaben in Statistisches Bundesamt, Fachserie 11, R. 4.2

Abb. 4: Endgültig nicht bestandene Abschlussprüfungen und Anteil der Notenstufe "sehr gut” im Jahr 2019.

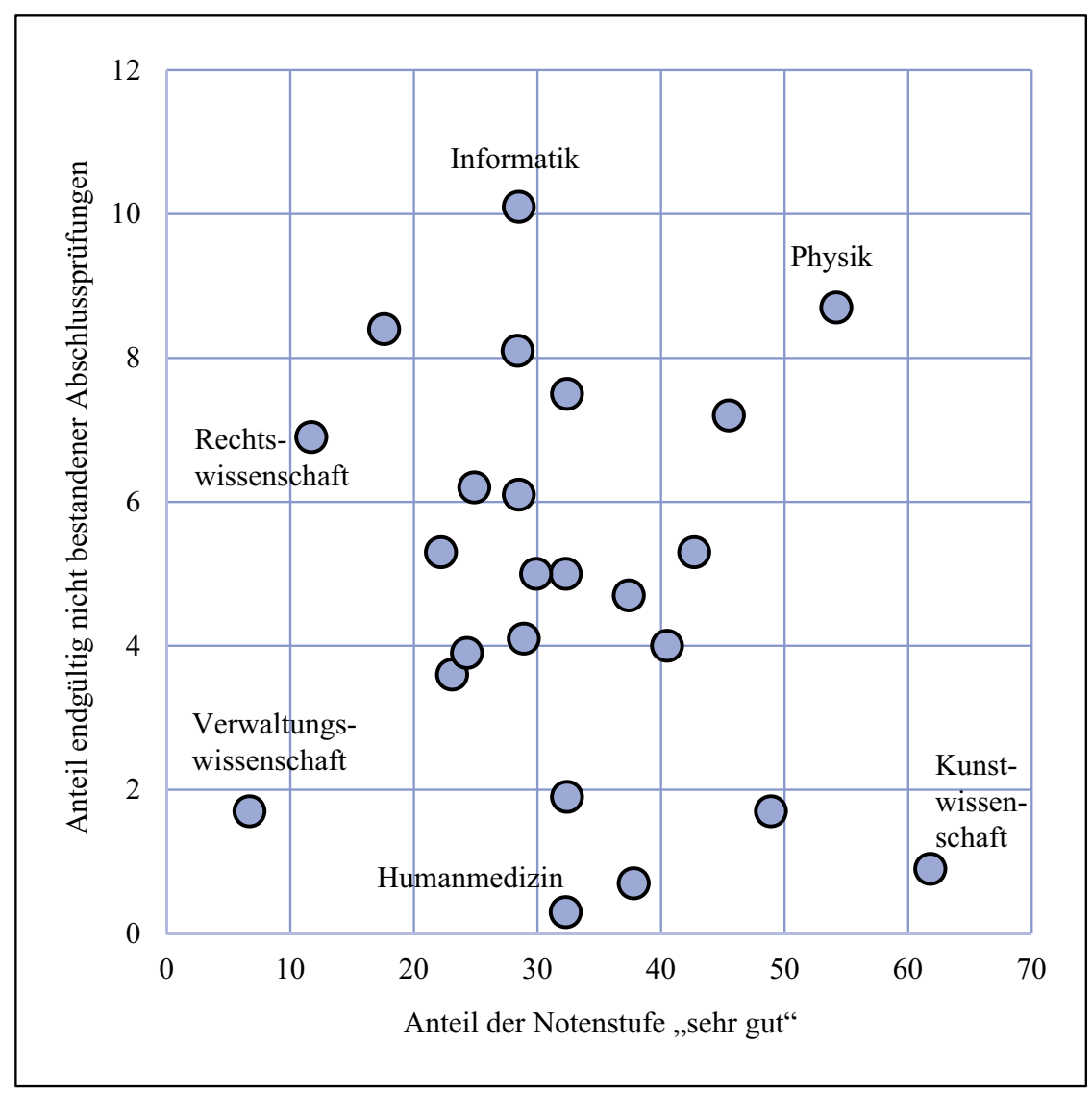


Durchschnitt aller erfassten Fächer ist sie von 2,9 \% im Jahr 2011 über 4,1 \% im Jahr 2015 auf 5,0 \% im Jahr 2019 gestiegen. Hohe Abbruchquoten weisen neben der Informatik die Physik, das Bauingenieurswesen und die Elektrotechnik auf, besonders niedrige neben der Kunstwissenschaft die Humanmedizin, das Sozialwesen und die Psychologie. Gelegentlich wird auch der Veränderung des Frauenanteils eine Erklärungsrolle zugedacht. Die Analyse der Daten nach Fächergruppen bestätigt dies nicht. Zwar nimmt der Anteil der Frauen an allen Studierenden immer noch zu, doch er hat inzwischen rund $50 \%$ erreicht, die Aufholprozesse sind also weitgehend abgeschlossen. Die häufig vertretene Annahme, Frauen würden in der Regel bessere Durchschnittsnoten erzielen, wird durch den hier verwendeten Datensatz nicht gestützt, die Anteile der "sehr gut"-Notenstufen unterscheiden sich zwischen Frauen und Männern in den einbezogenen Fächern nicht systematisch. Was sich dagegen systematisch unterscheidet, ist der Frauenanteil nach Fächern: Im Jahr 2019 betrug er 83,5\% in den Erziehungswissenschaften, $82 \%$ in der Germanistik und 81,4 \% im Sozialwesen auf der einen Seite und nur 20,8 \% in der Informatik, 20,7 \% im Maschinenbau und 13,4 \% in der Elektrotechnik auf der anderen Seite. Diese strukturelle Differenz in der Fächerwahl ist seit Jahren krass und ändert sich zudem nur sehr langsam, wenn überhaupt.

In ganz ähnlicher Weise verfestigt sind die festgestellten erheblichen Differenzen im Niveau der Notengebung nach Fächern. Dies legt die Vermutung nahe, dass tief eingeübte fachspezifische Benotungskulturen, die sich nur langsam ändern, ursächlich dafür sein dürften. Soweit Anpassungen zwischen den Fächern stattfinden, gehen sie in Richtung auf Angleichung via „Noteninflation“. Diese Grundentwicklung ist wie erwähnt bedenklich, da damit die Aussagekraft der Qualität von Studienabschlüssen leidet. Personalverantwortlichen ist diese Entwicklung natürlich nicht verborgen geblieben. Statt auf die Gesamtnote wird deshalb auf Nachkommastellen und Fachnoten im Studienverlauf geachtet. Die Hochschulen wären vor diesem Hintergrund gut beraten, ihre Benotungspraxis kritisch zu überprüfen. Bei den Zulassungsprozeduren zu Master-Studiengängen stehen sie vor den gleichen Problemen wie Personalverantwortliche: Wie verlässlich zeigen die Bachelor-Zeugnisse die Qualität der Bewerber an?

\section{Literatur}

Grözinger, G. u. Müller-Benedict, V. (Hrsg., 2017): Noten an Deutschlands Hochschulen. Analysen zur Vergleichbarkeit von Examensnoten 1960 bis 2013, VS Verlag für Sozialwissenschaften, Wiesbaden.

Wienert, H. (2018): Noteninflation - Aktuelle Tendenzen der Notengebung an deutschen Hochschulen. In: „WiSt - Wirtschaftswissenschaftliches Studium. Zeitschrift für Studium und Forschung“. 47. Jg. (2018), Heft 2-3, S. 65-69.

Statistisches Bundesamt (2020): Bildung und Kultur. Prüfungen an Hochschulen 2019 (Fachserie 11 Reihe 4.2). Wiesbaden. Daten für Jahre vor 2019 nach https://www.statistischebibliothek.de/mir/receive/DESerie_ mods_00000115 (Abruf 25.11.2020). 